\title{
Saúde das Trabalhadoras: O Caso da Lavanderia de uma Indústria Química de Cloro-Soda
}

\section{Female Workers' Health: The Case of a Laundry at a Sodium Chlorate Factory}

Jussara C. de Brito'; Ubirajara Mattos'

Vanda D'A. Soares' \& Heloisa P. Ferreira'

\section{BRITO, J. C.; MATTOS, U.; SOARES, V. D'A.; FERREIRA, H. P. Female Workers' Health: The Case of a Laundry at a Sodium Chlorate Factory. Cad. Saúde Públ., Rio de Janeiro, 11 (4): 543-551, Oct/Dec, 1995.}

This article presents a case study on working and health conditions of female workers and former workers in the laundry at a sodium chlorate plant. The women's main function was to wash uniforms of workmen who handled metallic mercury during production and maintenance. The aim of this study is to show the health effects of this work process by analyzing workplace conditions, evaluating clinical aspects in the women, and performing individual interviews in order to obtain more precise information based on their personal experience.

The authors demonstrate how harmful this type of work is to the women workers' health, posing a serious risk for them in particular and workers' conditions in general in the factory. They also point out that most of what is known about women's occupational activity (in terms of its contents and risks), in addition to their specific problems and different diseases, may be disguised by gender relationships.

Key words: Gender; Female Labor; Mercury; Chemical Industry; Workers' Health

\section{INTRODUÇÃO}

A questão que será tratada nesse artigo diz respeito ao trabalho feminino e suas condições, segundo uma proposta de afirmação da visão de gênero da saúde dos trabalhadores. A justificativa para essa abordagem é a relevância das diferenças vivenciadas por homens e mulheres no processo de trabalho, como resultado da divisão sexual do trabalho, uma vez que os perfis de morbi-mortalidade são determinados pela relação capital/trabalho, segundo as diferentes classes sociais e suas frações, bem como em função do sexo da força de trabalho (Brito \& D'Acri, 1991).

Centro de Estudos da Saúde do Trabalhador e Ecologia Humana, Escola Nacional de Saúde Pública/Fiocruz. Rua Leopoldo Bulhões, 1480, Rio de Janeiro, RJ, 21041-210, Brasil.
Para Scott (1989), o gênero é constitutivo das relações sociais e a primeira forma de significar as relações de poder. Para a autora o gênero é "um meio de decodificar o sentido e de compreender as relações complexas entre diversas formas de interação humana" (Scott, 1989: 17). Através da qualificação, que é construída pelas relações sociais de sexo na empresa e pela empresa, os gêneros são produzidos e reproduzidos (Kergoat, 1993).

A discussão de Verena Stolke (1980: 90) sobre a reprodução e o trabalho doméstico mostra os mecanismos de obscurecimento da subordinação das mulheres, que são mantidos mesmo no trabalho assalariado: "A ética do trabalho e do esforço pessoal, característica e central na sociedade burguesa, obscurece a especificidade da subordinação das mulheres. De acordo com esta ética quem trabalha são os homens, e apenas o trabalho produz; então são apenas os homens que participam ativamente do progresso da sociedade e merecem 
respeito e poder". Para Kergoat (1993), a literatura sobre o trabalho feminino coloca em evidência a importância extrema do trabalho assalariado para compreender a construção constante da identidade de gênero, ao longo da vida de cada indivíduo.

A organização do trabalho tem um papel fundamental na definição do conteúdo do trabalho e seus riscos. Em primeiro lugar, porque é através da forma pela qual o trabalho é organizado que são estabelecidas as tarefas, as máquinas e equipamentos a serem utilizados, os métodos, o tempo e ritmo de trabalho, a utilização de espaço, etc. Em segundo lugar, porque a organização do trabalho também define as relações entre os trabalhadores e entre trabalhadores e chefia. A organização do trabalho é uma peça importante para a construção do sentido do trabalho: enquanto possibilidade da expansão ou contração, enquanto sofrimento ou crescimento. Segundo Hirata \& Kergoat (1987) a organização técnica e social do trabalho difere segundo a predominância de homens ou de mulheres como operários de uma fábrica e, mais do que isso, as relações de força capital/trabalho não são a mesmas, segundo o trabalho se conjugue no feminino ou masculino.

Quanto à noção de risco para a saúde das trabalhadoras, essa deve ser pensada à luz das relações sociais de sexo. Nesse sentido é importante chamar atenção para o que foi apontado por Hirata \& Kergoat (1987), sobre a dificuldade das trabalhadoras reconhecerem problemas patogênicos que lhes são comuns, oriundos do processo de trabalho e de características biológicas, como conseqüência de não se perceberem como um grupo, um grupo gerado pelas relações sociais de sexo, um grupo sexuado. Refletir sobre os riscos para a saúde das trabalhadoras é refletir sobre as características das relações de trabalho específicas e sobre o conhecimento que as mulheres têm do seu trabalho: é construir um conceito próprio, que reflita a visão e os problemas delas.

Neste texto será colocado, especificamente, o problema das trabalhadoras da lavanderia de uma indústria de cloro-soda localizada no Rio de Janeiro. Essa empresa está sob fiscalização desde dezembro de 1990, quando o Programa de Saúde do Trabalhador, da Secretaria de Estado do Rio de Janeiro (PST/SES-RJ), recebeu uma denúncia do Sindicato dos Trabalhadores das Indústrias Químicas do Rio de Janeiro de que vários trabalhadores contaminados por mercúrio haviam sido demitidos.

Após um longo processo de negociação entre a Empresa, órgãos públicos e o Sindicato dos Trabalhadores, foi elaborado um Acordo para Mudança de Tecnologia e Defesa do Meio Ambiente - Controle do Mercúrio e do Cloro, firmado em maio de 1992 entre a Empresa, o Sindicato, a Assembléia Legislativa do Rio de Janeiro (Comissão de Ciência a Tecnologia), a Fundação Estadual de Engenharia e Meio Ambiente da Secretaria de Estado de Meio Ambiente e Projetos Especiais (FEEMA) e a Secretaria de Estado de Saúde do Rio de Janeiro (SES/ $\mathrm{RJ})$. A principal medida prevista no acordo é a substituição de todas as células de mercúrio, utilizadas nos setores de produção (as Eletrólises), para a fabricação dos produtos através de processo eletrolítico, por células de membrana, menos prejudiciais à saúde. No período de mudança, a ser realizada no prazo de 4 anos e meio, a empresa deverá eliminar ou controlar os riscos presentes nos locais de trabalho. A realização da tarefa de lavagem dos uniformes dos trabalhadores das áreas de contato direto com o mercúrio (Eletrólises e Manutenção), na lavanderia da empresa, foi mantida, a fim de evitar a contaminação de pessoas de fora da fábrica, consistindo-se assim numa "barreira" de proteção para essas pessoas, mas gerando uma situação de risco para as funcionárias. A substituição da tecnologia empregada implicará a eliminação do risco de contaminação por mercúrio, mas não necessariamente a melhoria das outras condições também prejudiciais, que serão descritas depois.

Nossa proposta, com esse estudo, é fazer emergir as questões relativas às trabalhadoras da lavanderia, do ponto de vista do processo de trabalho e dos riscos à saúde, numa perspectiva de revelação de seus problemas.

\section{METODOLOGIA}

Inscrita no campo da Saúde do Trabalhador, essa pesquisa é parte integrante de um projeto amplo de estudos sobre o trabalho e a saúde nas indústrias químicas do Rio de Ja- 
neiro, que está em desenvolvimento no Centro de Estudos de Saúde do Trabalhador e Ecologia Humana (Cesteh/Ensp/Fiocruz), que tem por objetivo a investigação das condições de saúde dos trabalhadores químicos, através de diversos enfoques complementares: um mapeamento de riscos, estudo de acidentes de trabalho e de riscos maiores a estudos de casos específicos, entre outros. Esse projeto se configurou a partir da demanda sindical, o que é um dado muito importante para a viabilidade do seu desenvolvimento e para o caráter dos resultados encontrados.

A Saúde do Trabalhador, como alternativa às antigas disciplinas que tratam da saúde e das condições de trabalho, busca a integração, através de uma abordagem multidisciplinar, com enfoques diferenciados e complementares. $\mathrm{O}$ conceito de Saúde do Trabalhador tem sido objeto de discussão na América Latina, no domínio da saúde pública, em países como México e Brasil, tendo em vista a reorganização do sistema de prevenção de doenças relacionadas ao trabalho. Em alguns países da Europa, como na Inglaterra e nos países escandinavos, é utilizada a noção de "Saúde no Trabalho", também como alternativa ao enfoque da "Medicina do Trabalho", que ainda ocupa um lugar privilegiado na França (Cassou, 1993).

No contexto do Brasil, dentro da discussão do Sistema Único de Saúde e devido à influência do Modelo Italiano, temos proposto métodos plurais e criativos para que seja possível o conhecimento das realidades locais e setoriais.

A conjugação de olhares e métodos distintos permite a apreensão do todo. Nessa perspectiva, utilizamo-nos nessa pesquisa, do estudo do processo e das condições de trabalho; da análise da atividade, no sentido dado pela ergonomia situada; da avaliação clínica e da análise da linguagem das trabalhadoras, através do material colhido em entrevistas.

O estudo das condições de trabalho proposto por Mattos \& Simoni (1982) parte da visão do trabalho enquanto espaço, energia e tempo. Do nível macro para o micro, pretende a identificação abrangente das condições de trabalho, da unidade de produção à seção e ao posto de trabalho, descrevendo a organização da empresa e do setor, o processo de fabricação, as energias e materiais empregados e o uso do tempo (jorna- das, ritmos de trabalho a pausas), com o objetivo de chegar a um diagnóstico. O mapeamento geral de risco da fábrica (Mattos et al., 1993) foi realizado através desse método, sendo feito o estudo das condições de trabalho da lavanderia, como um setor específico, para essa pesquisa, no primeiro semestre de 1993.

A ergonomia propõe a análise da atividade como método, tendo como procedimentos fundamentais a observação e o diálogo com os trabalhadores. A observação da atividade deve ser realizada por um período longo de tempo e em diferentes momentos. Segundo Teiger (1992), a compreensão da atividade de trabalho passa obrigatoriamente pela confrontação de conhecimentos heterogêneos e complementares, tanto dos trabalhadores quanto dos pesquisadores, podendo ser realizada a co-produção de uma representação original. A atividade de trabalho, enquanto objeto de pesquisa "tem características absolutamente originais, pois trata-se de um objeto que não é um dado, mas é um objeto a construir, a constituir e a reconstituir" (Teiger, 1992: 8). Segundo a autora, a atividade é apreendida pela confrontação das observações e dos modos operatórios diversos, cujos resultados são restituídos e discutidos com os trabalhadores, a pelas entrevistas individuais ou coletivas que visam à explicitação dos fenômenos observados e à expressão dos conhecimentos próprios dos trabalhadores sobre a situação. Assim, é "através do diálogo e pela confrontação dos pontos de vista que se constrói pouco a pouco a representação da atividade e de seus processos subjacentes e das conseqüências ressentidas pelos indivíduos sobre sua saúde e sua vida fora do trabalho" (Teiger, 1992: 8).

As entrevistas realizadas nesse estudo foram de dois tipos: diálogos livres feitos no próprio local de trabalho, com o objetivo de compreender o processo de trabalho, e entrevistas individuais, feitas na residência das trabalhadoras, utilizando um roteiro. Esse instrumento foi desenvolvido com a finalidade tanto de se obter informações objetivas e comparáveis, quanto de permitir a verbalização do conhecimento das trabalhadoras sobre a atividade e as interferências sobre a vida e a saúde. Foi pensado também como mecanismo viabilizador da construção coletiva desse conheci- 
mento. Foram ouvidas cinco trabalhadoras; três trabalhadoras antigas, demitidas durante a pesquisa, e duas trabalhadoras contratadas para substituir as primeiras, mas através de contrato por empreiteira.

\section{O TRABALHO NA LAVANDERIA E A SAÚDE DAS TRABALHADORAS}

O trabalho na lavanderia da fábrica consiste em lavar e passar os uniformes de aproximadamente 125 trabalhadores da empresa e da empreiteira, além de roupas da enfermaria (lençol, jaleco e toalhas de rosto), toalhas de mesa, toalhas de banho e peças do consultório do dentista.

Todos os uniformes a serem lavados são de trabalhadores que atuam diretamente em locais mais expostos ao mercúrio, ou seja, trabalhadores da Eletrólise e das Oficinas de Manutenção. Para isso, há uma listagem com os nomes desses trabalhadores, pois não devem ser lavadas roupas de pessoas de outros setores.

O tempo de trabalho das três mulheres que executavam essa atividade - as trabalhadoras antigas - variava de 2 a 6 anos. A nova equipe foi contratada em abril de 1993. A jornada de trabalho na lavanderia é de 7:30 às 16:30 horas. Até abril de 1993 havia alternadamente atividade aos sábados, no horário de 7:30 às 11:30 horas, para uma dupla. Após essa data, o trabalho aos sábados tornou-se regular.

As três primeiras foram contratadas como "Auxiliar de Serviços Gerais", enquanto que as outras (as atuais) têm o cargo de "Limpadoras". Ambas as denominações não caracterizam a profissão exercida por elas, cuja especialidade é a lavagem e o manuseio de peças contaminadas por mercúrio.

A mudança do tipo de vínculo, prestação de serviço de empresa contratada para atuar dentro da fábrica, implicou para as novas trabalhadoras um salário menor - menos que dois salários mínimos - além de perdas como a impossibilidade de se filiarem ao Sindicato mais combativo (dos químicos), o direito de almoçar no restaurante da empresa e o convênio médico. As ex-trabalhadoras recebiam mais que dois salários mínimos. Na carteira de trabalho das lavadeiras atuais não há o registro do adicional de insalubridade, embora elas o recebam.

$\mathrm{Na}$ lavanderia, o processo de trabalho se desenvolve da seguinte maneira. As roupas são levadas para lá, parte delas por uma pessoa responsável por isso a outra parte individualmente. São depositadas no chão (a maior parte) ou em uma bancada. Ao contrário do prescrito como uma medida de proteção, nem sempre as roupas são levadas em sacos plásticos, havendo constantemente o contato direto das trabalhadoras com elas. Após a lavagem, as roupas são retiradas das máquinas e colocadas numa centrifugadora, que se encontra relativamente próxima às outras. A fase seguinte é a secagem das roupas numa secadora, que está localizada a uns quatro metros de distância das outras máquinas. Já secas, as roupas são colocadas sobre um balcão e são dobradas para serem passadas numa prensa. Depois disso são acondicionadas em sacos plásticos (operação suprimida após a admissão das novas funcionárias), que são fechados em uma seladora e guardados nos escaninhos correspondentes. Os trabalhadores procuram suas roupas pelos números, que devem ser achados pelas lavadeiras. Essas fases do processo de trabalho são realizadas por meio de várias pequenas atividades, que compõem o todo, como a limpeza e secagem constante do chão, a colocação de mangueira na centrifugadora etc. Todo o transporte das roupas é feito manualmente, implicando grande esforço físico, principalmente quando as roupas estão molhadas. Apenas os uniformes são lavados em máquinas; as outras peças são lavadas em tanque. Os equipamentos utilizados são: uma secadora, uma passadeira (prensa), uma centrifugadora, duas lavadoras. Todos do tipo industrial, com excessão de uma lavadora. A centrifugadora oferece risco de acidentes, pois tem rotação bastante alta e, tanto a mangueira colocada para enxagüe, quanto partes de roupa que eventualmente ficam do lado de fora, em função do ritmo de trabalho e da inexistência de uma tampa que impediria a ocorrência disso, tornam-se verdadeiros chicotes, conforme registro das próprias lavadeiras.

\footnotetext{
"Ali tem duas máquinas perigosas. Até aconteceu um acidente com a Bela, com a
} 
menina que trabalha comigo. Ela colocou a roupa na centrifugadora, ficou a pontinha de uma camisa pra fora da boca da máquina. No que ela ligou aquilo deu umas lambadas nela, que ela ficou toda vermelha. Devido à velocidade dela”. (Ana)

A quantidade de roupas a serem lavadas varia muito, mas houve um acréscimo significativo desde que, com o Acordo, se proibiu que os trabalhadores almoçassem com roupas sujas. Por outro lado, fatores ligados à organização do trabalho, como a ausência de material para a lavagem ou uma maior exigência para os operários trocarem as roupas no meio do dia (almoço), interferem diretamente nessa produção, gerando a sobrecarga em determinados dias e a impossibilidade de desempenhar as funções nos outros. O que ocorre é que o trabalho é aparentemente organizado "informalmente", pois o controle sobre o que é feito, como é feito e o quanto é feito cabe às próprias lavadeiras, que fazem um registro da quantidade de peças lavadas diariamente.

\section{"Lá todo mundo fazia igual... Quem botava} mais a roupa na máquina era a Cida. E eu e a Eva é que passava mais. E a gente ajudava a botar na secadora. Porque assim ficava mais dividido". (Diva)

Ao mesmo tempo, nenhuma delas sabe bem quem é ou foi o chefe e como se dá o controle de seu trabalho. Seguem instruções gerais dadas na implantação da lavanderia por um técnico de segurança do trabalho. Essa questão é bem explicada pelo depoimento de uma delas:

"A gente parecia uma bola; todo mundo era chefe da gente. Depois do X, ultimamente ficou sendo o $Y$. Quando nós saímos de lá, era o $Y$. Depois disseram que era o $Z$, no começo”. (Diva)

Sentem-se esquecidas numa parte distante da fábrica, mas são totalmente responsáveis pelo que fazem, mesmo que isso não seja reconhecido e valorizado. Isso pode indicar o que a ergonomia já demonstrou, no que se refere à inexistência de atividades exclusivamente físicas, mesmo as mais simples, pois o componente psíquico-mental da atividade em questão se expressa pelo fato de que elas devem, de fato, coordenar todo o andamento do trabalho, controlar a qualidade do serviço realizado e responder por problemas eventuais que ocorram com as roupas.

Teoricamente, de alguns anos para cá, as roupas seriam levadas sempre em sacos plásticos fechados para evitar a contaminação. Há, porém, uma grande variabilidade nessa operação; como já foi dito anteriormente, o transporte das roupas é feito tanto pelo funcionário responsável, em carrinho, quanto pelos operários, uma a uma, raramente em sacos plásticos.

O trabalho descrito como repetitivo, além de exigir um esforço físico significativo e da possibilidade de exposição ao mercúrio metálico, é realizado sob ruído e calor intenso. O ambiente externo, como a experiência de Eva comprova, contamina o espaço interno da lavanderia.

"É o tempo todo a mesma coisa. Você dobra a roupa, bota na máquina. Aí, fecha a máquina, passa. Tem que pegar, virar novamente a roupa do outro lado. É o mesmo movimento toda hora". (Ana)

"Tinha hora que a gente tinha que pular em cima do pedal, pra poder trancar a máquina. Se não trancar não passa...”. (Eva)

"Lá é uma poeirada danada. Tinha um jato de areia, que ainda era pior. Não podia nem abrir a boca, que vinha aquela poeira”. (Eva)

“Aquele exaustor nem adianta, ele faz mais barulho do que tira a temperatura quente de lá. Aí eles resolveram abrir aquele janelão, mas também quando ligava a máquina de passar, que é a vapor, e a secadora, não adiantava muito. No verão era uma tristeza”. (Eva)

A lavanderia está instalada em um prédio que se situa entre a oficina elétrica e de instrumentação e a sala de jateamento de areia, numa área relativamente distante da entrada da fá- 
brica, próxima às oficinas de manutenção. Sua construção antiga é de um único pavimento, com pé-direito variando entre 4 e $5,5 \mathrm{~m}$. A cobertura é de duas águas, com estrutura de madeira e telhas de cimento amianto. As paredes são construídas em alvenaria e pintadas de branco. O piso, na cor cinza, é revestido com resina do tipo epox. Ele apresenta irregularidades e o seu caimento favorece poças e acúmulo de água, principalmente nas áreas de circulação.

Observa-se que o arranjo físico da lavanderia não obedece ao fluxo do processo de trabalho, pois a disposição dos equipamentos e armários provocam a movimentação excessiva das trabalhadoras, no transporte manual das roupas que, como já foi dito, representa uma carga de trabalho física relevante. Há também máquinas desativadas, que ocupam espaço. Os materiais de limpeza são estocados num canto, ao chão ou sobre a lavadora sem uso, sem condições adequadas.

A sala só possui uma porta (com $2 \mathrm{~m}$ de largura) de acesso para uma rua de terra. As áreas de circulação apresentam distâncias variando entre $0,55 \mathrm{~m}$ a $1,10 \mathrm{~m}$. Como referência, há os valores estabelecidos pelas NR12 (Normas Regulamentadoras em Segurança e Medicina do Trabalho), que dispõem sobre máquinas e equipamentos a que estabelecem o mínimo de $1,20 \mathrm{~m}$ para as vias de circulação e para as que levam às saídas (MTb, 1993). Isso significa que as condições mínimas de conforto e segurança não são encontradas nesse espaço de trabalho.

Quanto aos índices ambientais, foram realizadas medições de ruído (Nível de Pressão Sonora-NPS), iluminação, temperatura do ar, ventilação e concentração de mercúrio no ar, em diversos pontos da lavanderia. Através dessa amostra podemos avaliar que as temperaturas no local são elevadas (entre 23,7 a $26,4^{\circ} \mathrm{C}$ ) considerando-se as condições do dia (nublado, com temperatura do ar entre 14 a $26^{\circ} \mathrm{C}$ a ventilação e temperatura externa ao prédio, às 11 horas, de $0,70 \mathrm{~m} / \mathrm{s}$ a $22,5^{\circ} \mathrm{C}$ ). As fontes de calor radiante (na secadora e na passadeira) são significativas e responsáveis pelo calor, juntamente com a convecção no local, gerando assim uma sensação térmica de desconforto. Somam-se ainda a esses equipamentos, as taxas de metabolismo das trabalhadoras durante a realização da atividade (moderada). A venti- lação natural é insuficiente $(0,1$ a $0,2 \mathrm{~m} / \mathrm{s})$, devido às características da construção (principalmente a cobertura e dimensões das janelas) e a localização do prédio no terreno exige a utilização de ventilação forçada e exaustão para renovação do ar e redução da temperatura no ambiente (MTb, 1993).

O nível de ruído (entre 78 a $81 \mathrm{~dB}(\mathrm{~A})$ - ruído contínuo - e 80dB (C) - ruído de impacto), apesar de não ultrapassar os limites de tolerância estabelecidos no Anexo 1 da NR-15 (atividades e operações insalubres) é elevado (MTb, 1993). Constata-se também a presença de vapor de mercúrio(Hg) no ambiente, em concentrações abaixo dos limites de tolerância estabelecidos no Anexo 11 da NR-15 - 0,04 mg/m $\mathrm{m}^{3}$ (MTb, 1993).

O nível de iluminação total da sala (artificial e natural) mostrou-se suficiente para a realização das atividades (entre 200 a 700 lux; medição feita com todas as lâmpadas acesas).

Como previsto no Acordo já citado, as antigas trabalhadoras foram atendidas no Ambulatório do Sindicato dos Trabalhadores Químicos-RJ, em junho de 1993 quando foi feita uma anamnese ocupacional, seguida de uma avaliação clínica, com ênfase no exame neurológico, além dos resultados de dosagens de $\mathrm{Hg}-\mathrm{U}$, realizadas pela empresa e pela Fundação Oswaldo Cruz (Fiocruz) em 1992 e 1993. Os sinais/sintomas apresentados pelas trabalhadoras sugerem um quadro de intoxicação mercurial (Vroom \& Greer, 1972), principalmente quando comparados com as resoluções publicadas pelas Secretarias de Saúde de São Paulo (Suds/Inamps-SP, 1991) e do Rio de Janeiro (Ses/RJ, 1993), que estabelecem critérios diagnósticos em casos de exposição ocupacional aos vapores de mercúrio metálico (Tabela 1 ).

Também não foram observados, durante a anamnese, dados da história patológica pregressa e familiar que justificassem este quadro, assim como etilismo e outras patologias que pudessem ser associadas.

Para a confirmação diagnóstica do hidrargirismo seriam necessários exames complementares, de acordo com o quadro clínico específico, assim como avaliação psicológica, seguida de testes psicológicos, que não foram realizados, devido a problemas internos do sindicato a dificuldades das próprias trabalhadoras. 
TABELA 1. Distribuição dos Sinais/Sintomas Apresentados Pelas Trabalhadoras da Lavanderia

\begin{tabular}{lc|c|c}
\hline & \multicolumn{3}{c}{ Trabalhadora } \\
\cline { 2 - 4 } Sinais/Sintomas & A & B & C \\
\hline 1. Gengivite & + & & \\
2. Úlceras Orais & + & & \\
3. Sialorréia & + & & \\
4. Tremor & + & & \\
5. Irritabilidade & + & + & + \\
6. Nervosismo & + & + & + \\
7. Fadiga & + & + & + \\
8. Distúrbios da Memória & + & & + \\
9. Tontura & + & + & + \\
10. Distúrbios do Sono & + & + & + \\
11. Mioclonia & + & + & + \\
12. Câimbra & + & + & + \\
13. Cefaléia & + & & \\
\hline \hline
\end{tabular}

No entanto, a complexidade da questão da saúde é transmitida pelo conjunto de observações, pois cada trabalhadora mostra um aspecto diferente do processo de adoecimento. $\mathrm{O}$ primeiro desses aspectos diz respeito à desigualdade nos cuidados dirigidos a elas, pelo pessoal da empresa, em comparação aos prestados aos trabalhadores do sexo masculino:

\section{"Nossos exames não eram feitos}

periodicamente não, antigamente não. De 92

pra cá é que começou, todo mês, eles chamarem a gente pra fazer exame. Porque antes não era assim não. A Dona Cida sempre falava: a lavanderia é esquecida, nem exame prá gente eles não fazem. Nós comentávamos entre nós mesmo: todo mundo faz exame, menos a gente. Levava tempo pra gente fazer um exame. A gente não sabia nem o resultado". (Eva)

As questões da saúde reprodutiva e da sexualidade são também emergentes e indicativas dessa complexidade.

"Quase eu morri com o G. Passei mal a gravidez. do começo ao fim, minha filha: pressão alta, problemas de nervos. Eu tomava era calmante e remédio para pressão. Já pensou, com 35 anos esperando menino? Ave-Maria! Eu tive uma inflamação nos rins que só faltei morrer". (Bela)
"Te digo mesmo: eu só arrumei outro homem, sem ser meu marido, pra mim, foi por causa de um médico; porque o médico achava que os meus problemas mais era porque eu fiquei sem homem muito cedo; eu era muito nova quando o meu marido deixou de mim". (Cida)

Da primeira equipe para a segunda houve uma mudança no que diz respeito ao nível de informação sobre mercúrio, em conseqüência do Acordo. As primeiras trabalhadoras só ficaram sabendo que trabalhavam com roupa contaminada durante esse processo de intervenção, pelos próprios trabalhadores. As outras receberam informações básicas na contratação, porém a dúvida e o medo persistiram.

"Sabia só que era roupa do pessoal que trabalha. Depois o pessoal ia lá e dizia - aí trabalha com mercúrio, é perigoso à beça; se a mulher pegar isso, vai andar até de perna aberta; cuidado com o mercúrio que a gente aqui já não está prestando prá nada”. (Diva)

"No princípio eu fiquei com medo desse negócio de mercúrio que tem ali. Logo no princípio. Mas depois tem uma moça que mora ali que trabalhou lá cinco anos e meio e a mulher está viva, é gorda...”. (Bela)

Outra dimensão da questão saúde se refere aos movimentos individuais no sentido da pro- 
teção. Como o risco de exposição ao mercúrio é para essas mulheres algo invisível, misterioso e incompreensível, e como o nível de segurança não lhes parece adequado, é necessário que sejam criados por elas próprias os mecanismos de proteção, como o citado por Bela:

\section{"Aqueles dois paus que eu mandei fazer,} que eu te mostrei lá. Pedi um homem para adaptar; aí ele arranjou um e aí passou lá no carpinteiro. E depois eu pedi outro, pra tirar aquela roupa quente, pra não chegar muito junto daquele vapor. Um é pra a gente colocar a roupa, pra não ter muito contato com aquela água, a gente soca assim com aquele pau. E o outro é pra gente não chegar muito perto daquele vapor, quando abre ali, prá tirar a roupa que está secando".

\section{CONCLUSÕES}

Com esse estudo de caso procuramos evidenciar os problemas específicos que afetam as trabalhadoras de uma indústria do setor químico, no que se refere à saúde. Investigando o processo de trabalho, as características ambientais, analisando a atividade que é desenvolvida, reunindo dados clínicos ocupacionais, considerando vida e trabalho como uma unidade inseparável e conjugando a visão das mulheres, podemos afirmar o que se segue.

A saúde dessas trabalhadoras, sob o ponto de vista do coletivo, é atingida, não só pelas condições materiais inadequadas, que as expõem a riscos particulares, mas também por um conjunto de fatores ligados à organização do trabalho, que as colocam numa posição desfavorecida, precária, com menos controle sobre sua saúde e menor possibilidades de reivindicar, questões geradas pela identidade de gênero.

Esse estudo mostra o que ocorre com um pequeno universo de trabalhadoras, em que condições são admitidas e em que condições são demitidas. São admitidas como trabalhadoras desqualificadas, sendo seu saber considerado natural e acabam se sentindo esquecidas num canto da fábrica. Seus problemas específicos, suas dificuldades e riscos não são valorizadas. São expulsas no momento em que têm a saúde mais deteriorada e são impedidas de conhecer melhor esse estado, pois a demissão, ao provocar o afastamento delas do conjunto dos trabalhadores da fábrica e do sindicato e ao impelí-las a se ocuparem com outras atividades remuneradas, impede o acompanhamento e o diagnóstico dos seus problemas de saúde. Por não terem suas qualificações reconhecidas e, assim, menor acesso aos postos de trabalho; ao mesmo tempo que são indispensáveis na economia familiar, relegam a saúde ao segundo plano: nessas condições ter um salário, mesmo que insuficiente, é o mais importante.

\section{RESUMO}

BRITO, J. C.; MATTOS, U.; SOARES, V. D'A.; FERREIRA, H. P.Saúde das Trabalhadoras: $O$ Caso da Lavanderia de uma Indústria Química de Cloro-Soda. Cad. Saúde Públ., Rio de Janeiro, 11 (4): 543-551, out/dez, 1995.

Esse artigo apresenta um estudo de caso sobre as condições de trabalho e saúde de trabalhadoras a ex-trabalhadoras da lavanderia de uma indústria do setor químico. A função principal dessas mulheres é lavar as roupas dos operários das áreas de produção e de manutenção que manipulam com mercúrio metálico, constituindo-se em áreas de risco. Nosso objetivo é mostrar como as mulheres são atingidas nesse processo de trabalho. Para isso realizamos uma análise do processo de trabalho e das condições ambientais da lavanderia, uma avaliação clínica das trabalhadoras, bem como entrevistas individuais com as mesmas, a fim de melhor apreender o objeto da pesquisa, através da linguagem das entrevistadas. As avaliações e análises nos permitiram verificar que esse trabalho é prejudicial à saúde, tanto pela história de vida de cada uma, como também pelas condições atuais em que é desenvolvido na empresa. Com esse artigo nos propomos também mostrar que os conhecimentos sobre a atividade (o conteúdo e os riscos) que essas mulheres realizam, sobre seus problemas específicos e sobre suas formas 
de adoecimento tendem a ser dissimulados por um conjunto de fatores afetos às relações de gênero.

Palavras-Chave: Gênero; Trabalho Feminino; Mercúrio; Indústria Química; Saúde do Trabalhador.

\section{REFERÊNCIAS BIBLIOGRÁFICAS}

CASSOU, B., 1993. Santé publique et santé au travail. Cahier du S.M.T.: Santé Publique et Médecine du Travail, 6: 15-16.

BRITO, J. \& D'ACRI, V., 1991. Referencial de análise para estudo da relação mulher, trabalho e saúde. Cadernos de Saúde Pública, 7: 201-214.

HIRATA, H. \& KERGOAT, D., 1987. Divisão Sexual e Psicopatologia do Trabalho. Trabalho apresentado ao IX Encontro Anual da ANPOCS, Águas de São Pedro. (Mimeo.)

KERGOAT, D., 1993. La qualification: un enjeu de rapports sociaux de sexe? Cahier du GEDISST/ IRESCO/CNRS, 7: 07-12.

MATTOS, U.; FREITAS, N. \& GONÇALVES, C., 1993. Indústria de Cloro-Soda do Rio de Janeiro: Levantamento e Diagnóstico das Condições de Trabalho. Relatório de Pesquisa, Rio de Janeiro: Cesteh/Fiocruz. (Mimeo.)

MATTOS, U. \& SIMONI, M., 1982. Roteiro de Projeto em Higiene e Segurança do Trabalho. São Carlos: DEP/Universidade Federal de São Carlos.
MTb (Ministério do Trabalho), 1993. Normas Regulamentadoras em Segurança e Medicina do Trabalho - Portaria 3214 de 08/06/78 MTb. São Paulo: Atlas.

SCOTT, J., 1989. Gênero: uma Categoria útil para Análise Histórica. Recife: SOS Corpo.

STOLKE, V., 1980. Mulheres e trabalho. Estudos CEBRAP, 26: 81-117.

SUDS (Serviço Unificado e Descentralizado de Saúde)/ INAMPS-SP (Instituto Nacional de Assistência e Previdência Social) 1991. Critérios para caracterização do Nexo Ocupacional e do Diagnóstico de Intoxicação pelo Mercúrio Metálico. Resolução SS-307 de 14.08.91. São Paulo: INAMPS/SP. (Mimeo.).

SES/RJ (Secretaria de Saúde do Estado do Rio de Janeiro), 1993. Critérios Diagnósticos, Orientação Clínica e Procedimentos de Notificação por Parte das Empresas nos Casos de Intoxicação pelo Mercúrio. Resolução no 838/SES, 30/03/93. Rio de Janeiro: SES/RJ. (Mimeo.)

TEIGER, C., 1992. Le Travail, cet Obscur Objet de L'Ergonomie. Colloque Interdisciplinaire Travail: Recherche et Prospective. Lyon: PIRTTEMCNRS. (Mimeo.)

VROOM, F. Q. \& GREER, M., 1972. Mercury vapour intoxication. Brain, 92: 305-318. 